\title{
EL PAPEL DEL ESTADO EN EL ORIGEN DE LAS ACTIVIDADES ECONÓMICAS EN UNA REGIÓN PERIFÉRICA DE COLOMBIA: ANÁLISIS COMPARATIVO ENTRE LA MINERİA Y LA AGROINDUSTRIA
}

\section{THE ROLE OF THE STATE IN THE ORIGIN OF ECONOMIC ACTIVITIES IN A PERIPHERAL REGION OF COLOMBIA: COMPARATIVE ANALYSIS BETWEEN MINING AND AGRO-INDUSTRY}

\author{
Juan Manuel Andrade Navia*
}

\begin{abstract}
Resumen
La evolución de actividades económicas, impulsadas por agentes empresariales, se constituye en objeto de estudio para comprender en toda su expresión, la realidad actual de las regiones. A la par, el apoyo que estructural o coyunturalmente puedan generar las instituciones del Estado se convierte en un elemento más en el estudio del surgimiento y desarrollo de las actividades que dinamizan la economía. Es en esta lógica que el presente estudio procura identificar y comprender el papel del Estado en el origen de las actividades económicas, para el caso particular de la minería y la agroindustria, en el departamento del Huila (región periférica de Colombia). Se pretendió comprender el fenómeno mediante la identificación y caracterización de las actividades económicas y sus relaciones con el Estado.
\end{abstract}

En efecto se determinó que el Estado juega un papel determinante en el surgimiento y consolidación de actividades económicas o en su defecto en las condiciones en las que históricamente se encuentran. En efecto, se aprecia un estímulo a las actividades mediante la financiación de estudios técnicos que permitiesen determinar las potencialidades del sector en el que se encuentran inmersas. Sin embargo, su rol en ocasiones se limita a labores de control y/o supervisión con pocos esfuerzos para su desarrollo.

Palabras clave: Estado, actividades económicas, minería, agroindustria, priorización productiva del sector con alto potencial, región periférica.

\section{Abstract}

The evolution of economic activities driven by corporate actors constitutes an object of study to understand, in all its expressions, the current reality of the regions. At the same time, the structural or circumstantial support that the state institutions can generate becomes an element in the study of the emergence and development of activities that stimulate the economy. It is under this logic that the present study seeks to identify and understand the role of the State in the emergence of economic activities, for the particular case of the mining and agribusiness in the department of Huila (a peripheral region of Colombia). It was intended to understand the phenomenon through the identification and characterization of economic activities and their relationships with the State. It was confirmed that indeed the State plays a leading role

Artículo recibido: 17/07/2013 Aprobado: 31/08/2013

* Joven Investigador Universidad Surcolombiana. Email: Juanmanuel.andrade@usco.edu.co 
in the emergence and consolidation of economic activities, or otherwise, in determining the conditions under which these activities are operating. It is noted that in fact, activities receive a stimulus through the financing of technical studies for assessing the potential of the sector in which they are immersed. However, its role is limited to control and / or supervision work, with little effort to boost its development.

Key words: State, economic activities, mining, agribusiness, prioritized productive sector with high potential, peripheral region.

\section{Aspectos teóricos}

El presente estudio abordó el papel del Estado y su influencia en el surgimiento de las actividades económicas minera y agroindustrial en la región periférica del sur de Colombia (el departamento del Huila), y sustenta sus conclusiones con un análisis comparativo de las variables más relevantes.

Con respecto a los estudios del papel de la institucionalidad en el surgimiento y desarrollo de actividades económicas, recientes investigaciones sobre los países en desarrollo han comenzado a salvar esta brecha, llegando cada vez más a la conclusión de que las relaciones entre las empresas y el Estado son decisivas para explicar los patrones de desarrollo vigentes a fines del siglo XX (Schneider, 1999).

Lo anterior podría estar directamente relacionado con la fragilidad de algunas economías de países en vías de desarrollo o subdesarrollados, donde la intervención y gestión del Estado crea oportunidades, que de otra manera el mercado no hubiese ofrecido de manera natural y que las empresas no hubiesen podido aprovechar por su propia gestión.

Así mismo, Doner (1991) afirma que «las diferencias en las relaciones entre las empresas y el Estado constituyen la explicación más importante de la variación entre países en lo conexo a su desempeño en las negociaciones sectoriales» (págs. 4-5).

Esto refuerza la idea de que la diferencia entre los estados de desarrollo en que se encuentren las actividades económicas en los diferentes países, se define en gran medida en el nivel de relación entre la gestión de las empresas privadas y las instituciones del Estado.

Para el caso colombiano, Thorp (1991) llegó a la conclusión de que la política económica había sido permanentemente más eficaz en Colombia que en Perú, y una de las razones principales de ello era que en Colombia la relación entre el estado y el sector privado «se caracterizó por un mayor respeto y confianza mutua» (pág. 195).

Algunas empresas, con campo de gestión en actividades o sectores estratégicos en el país, generaron estrechas relaciones con el Estado, a través de los vínculos personales entre empresarios y figuras políticas, que a futuro les ofreció excelentes beneficios.

Por ejemplo, para el caso latinoamericano, el crecimiento sostenido de Chile después de 1985 debió mucho a las estrechas relaciones entre las empresas y el Estado, como ocurrió en los países asiáticos de desarrollo exitoso (Schneider, 1999:17).

El punto de partida, según Evans (1995), es la intervención del Estado como un elemento indispensable de la rápida industrialización tardía de algunos países latinoamericanos y del sudeste asiático, especialmente en Corea, que es el principal referente empírico de la autonomía enraizada (embeddedautonomy).

Siguiendo la venerable tradición de Gerschenkron (1962), Evans especifica además los roles y funciones particulares que asume el Estado en las sucesivas etapas de la industrialización, denotando un estrecho vínculo a lo largo de las diferentes etapas en el proceso de fortalecimiento de actividades económicas y empresariales.

Los roles que mejor promueven la transformación industrial dependen de las características de cada sector, sobre todo de la forma en que cada uno de ellos está estructurado internamente, y del modo en que se combinan diversos roles del Estado (pág. 14).

Si Evans califica la autonomía como «enraizada» es para destacar que los burócratas eficaces tienen relaciones muy estrechas con las empresas. Para medir el grado de enraizamiento se tiene en cuenta qué parte de su tiempo pasan los burócratas con los empresarios, así como la 
densidad de las redes más formales que los vinculan con estos.

Existen muchos ejemplos claros donde la clase empresarial se encuentra ligada a la clase política. Para el caso latinoamericano el fenómeno predomina con mayor fuerza en las esferas locales y regionales, dado la limitación del alcance de su gestión empresarial por el amplio número de pequeñas y medianas empresas. No obstante, los grandes emporios y grupos empresariales ejercen fuerte poder sobre la política nacional y sus destinos.

Desde la perspectiva teórica de la economía institucional, se considera a la empresa como una organización más, dentro de las disponibles, para la asignación de recursos. Da una nueva visión para abordar los problemas microeconómicos tradicionales como son los mercados imperfectos, la financiación de las empresas y la internalización del mercado de trabajo (Ibíd.).

Dentro del enfoque el Estado juega un papel crucial en el modelo. North y Thomas (1973) señalan que los gobiernos asumen la protección y aplicación de los derechos de propiedad porque ellos pueden hacerlo a costos menores que los grupos privados que podrían organizarse voluntariamente. Sin embargo, los gobiernos, apremiados por sus necesidades fiscales, pueden establecer ciertos derechos de propiedad que obstaculicen el crecimiento en vez de alentarlo: no hay garantías de que los arreglos institucionales productivos sean los que se establezcan.

Además, establece que el conocimiento y la tecnología son variables a tener en cuenta en el desarrollo de la sociedad, y en la realidad la estructura de la organización política y económica (las instituciones), quienes determinan el desarrollo económico, incluyendo el nivel de conocimientos y avances tecnológicos (North, 1987).

El papel del Estado, como institución, permite definir otro tipo de relaciones e iniciativas, tanto sociales y económicas como empresariales. La expedición de normas, códigos, políticas y el apoyo a grupos de interés marcan la pauta en el desarrollo. No obstante, a los lugares 0 situaciones a las que no llega el Estado, o lo hace muy débilmente, su vacío es cubierto o será cubierto por otras instituciones (Valdaliso et al., 2000).

En este sentido, el enfoque institucional se entiende como el conjunto de reglas y normas, que pueden ser for- males 0 informales, y que relacionan los sistemas sociales y económicos en múltiples aspectos, tales como las interacciones sociales, las expectativas sobre los beneficios a partir de las transacciones económicas y las formas en las cuales los actores determinan sus posibilidades de acción, de acuerdo con sus intereses individuales (Matzavinos et al., 2004, North, 1981, North, 1990).

Las instituciones se pueden entender entonces como reglas formales e informales, sociales y económicas que están presentes en sistemas con diferentes niveles (local, regional, nacional), comprendidos y posiblemente cumplidos por los actores involucrados, y las cuales permiten que el sistema social evolucione, en la medida en la que el contexto en el cual están inmersas se vea afectado o modificado (Granovetter, 1985, Aggarwal y Dupont, 1999, Folke et al., 1998, Ostrom, 1990, Ostrom, 2003, Swallow et al., 2006).

Las instituciones tienen como estandarte las estructuras sociales, donde son aspectos claves la información, las escalas de castigo y reconocimiento y la confianza entre los actores (Granovetter, 2005).

Así mismo, Valdaliso y López (2000) reconocen el papel de la legislación y la normatividad como un determinante dentro de la génesis y evolución de las empresas y los sectores industriales, especialmente, lo concerniente al marco legal que permitiese la asociación de capitales que posteriormente pudiesen invertirse.

Desde otra perspectiva, Valdaliso y López (2000) presuponen que las razones que contribuyeron al surgimiento de la empresa se tienen que buscar en la ampliación de las oportunidades de negocio consecuencia del crecimiento de los mercados y un cambio tecnológico, en un marco institucional favorable a la iniciativa individual y a la propiedad privada.

\section{Metodología}

La investigación se enmarcó en la línea de investigación de historia empresarial, que tal como se reseñó anteriormente, para el caso colombiano, ha sido abordado desde perspectivas y con metodología diferentes pero aún se encuentra muy superficial. En consecuencia, se constituye en una investigación de tipo exploratorio, en la medida en que se erige como la primera aproximación del investigador al objeto de estudio. 
Esta investigación permitió por una parte, llevar a cabo una recopilación de tipo teórico y, de otra, seleccionar algunos fenómenos de interés para la investigación, acordes con la definición de empresas y Estado asumidos en el marco de referencia, para con ellos efectuar la identificación de las variables relevantes.

El procedimiento lógico a seguir para la adquisición de conocimiento fue el método inductivo, por cuanto el fenómeno del origen de las actividades y el papel del Estado se abordaron de manera particular, de acuerdo a los diferentes documentos primarios y secundarios relacionados con el objeto de estudio.

Es en esta lógica que el presente estudio procuró identificar y comprender el papel del Estado en el origen de las actividades mineras y agroindustriales en el departamento del Huila. Se pretendió comprender el fenómeno mediante la identificación y caracterización de las diferentes acciones adelantadas por el Estado en las actividades económicas propuestas.

Para el desarrollo de la investigación se recurrió a documentos como archivos de las empresas y archivos de prensa. Igualmente, a las fuentes orales de los sujetos que se encuentran inmersos en la actividad económica. En particular, se espera que la información primaria, así como la secundaria permita descubrir las estructuras significativas que explican el origen de las actividades objeto de estudio.

En consecuencia, se utilizaron como fuentes secundarias publicaciones de investigaciones sobre casos colombianos y estudios teóricos conceptuales sobre tópicos en cuestión, los cuales se revisaron con el objeto de apropiar mayores elementos metodológicos para la realización del trabajo de campo.

Para la recolección de información se utilizaron las entrevistas semi-estructuradas, con el propósito fundamental de conformar testimonios y reconstruir los hechos, y el análisis de fuentes documentales examinando el significado y sentido del mensaje estudiado.

\section{Hallazgos}

A la luz del institucionalismo desarrollado por North (1973), el papel del Estado en el avance de la actividad minera, en el departamento del Huila, cumple una doble función: primero, como promotor de estudios prelimina- res de caracterización e inventarios mineros y segundo como obstaculizador, en tanto desarrolla una legislación altamente restrictiva para el desarrollo de la misma. Los trámites para la adjudicación de las licencias de exploración y explotación del mineral, se constituyen en grandes obstáculos para su adelanto.

No obstante, los abultados trámites que se perciben como consecuencia de la normatividad expedida, que generan efectos negativos para el mediano y pequeño minero, no tienen el mismo efecto para la gran minería. Lo anterior como consecuencia del nuevo código de minas, sancionado con la Ley 1382 de 2010, en el cual se eliminan estas categorías y se reconoce únicamente la explotación a gran escala, con la agravante de que con una sola categoría, todos los mineros, grandes y pequeños, son iguales y, en consecuencia, deben presentar las mismas condiciones ante el Estado.

En este sentido, de acuerdo con Valdaliso et al. (2000), el papel del Estado, en este caso el Estado colombiano, redefinió las estructuras sociales y productivas, en el sector minero huilense, especialmente para la actividad minera del departamento. Lo anterior a partir de los aspectos relacionados con la expedición de licencias de explotación, que generó un fuerte movimiento de minería de hecho o tradicional.

En este sentido, el Estado como institución generadora de reglas y normativas que alientan el desarrollo de actividades económicas (Valdaliso et al., 2000), está enfocando sus esfuerzos, a partir de lo que se puede intuir con las últimas reformas al código y los excesivos trámites para la adquisición de explosivos, al estímulo para el ingreso de grandes empresas mineras, por lo general, de origen extranjero a la actividad minera. Esto explicado, en alguna medida, por las grandes inversiones en tecnología que se requiere para su explotación.

Dichas situaciones generaron un efecto desalentador en los esfuerzos que se venían realizando hasta el momento. El desinterés y abandono por parte del Estado permitió, como afirma Valdaliso et al. (2000), el surgimiento de otras instituciones que asumieran su rol; por un lado, la génesis de organizaciones como las asociaciones locales, de carácter formal, que buscaron apoyar procesos de desarrollo en los referente a lo técnico y tecnológico; y de otra parte, el nacimiento de grupos, de tipo informal, que permiten el apoyo a las personas que lo requieren. Estas últimas tienen su justificación en las relaciones familiares y de parentesco. 
A pesar de lo expresado, es preciso aclarar que durante las décadas de 1950 y 1960, el Estado, a través del Ingeominas y del Ministerio de Minas y Energía, auspició la ejecución de por lo menos 5 estudios de caracterización e inventarios mineros en el departamento.

Dicha situación no se volvió a repetir en las últimas décadas a pesar de los esfuerzos por la realización de un gran estudio de cuantificación de reservas minerales. Al respecto, don Rodrigo Perdomo, durante su estancia como secretario del Senado, logró que por parte del Ministerio surgiera un proyecto de cuantificación de reservas de mármol en el Distrito Minero Noroccidental (zona de Palermo), iniciativa que se abortó al advertirse a don Rodrigo como directo beneficiario, con una licencia de explotación vigente sobre más de 100 hectáreas de terreno en la zona de la Lupa.

Caso contrario sucede con otras actividades económicas en el departamento. Para el caso de la actividad agroindustrial, el apoyo del Estado como fuente de recursos y sustento tecnológico, además de estimular su organización, permitió su consolidación desde la conformación de redes de apoyo entre las empresas más notables del entorno.

Esfuerzos que para este caso, se realizaron a inicio de la década de 1990, con el diseño de proyectos para la piscicultura, que se entregaron o presentaron a inversionistas para su ejecución. Además de apoyos a la consecución de técnicos o expertos para la asesoría a la actividad.

Para el caso de la actividad agroindustrial, el Estado a través de instituciones locales como la Secretaría de Agricultura y Minería, desde la década de 1990 propició estudios de factibilidad para la promoción de la actividad agroindustrial.

Las instituciones de carácter nacional tuvieron poco protagonismo en una primera etapa de surgimiento, en el sentido estricto de una actividad generadora de resulta- dos económicos que trasponga el carácter de subsistencia familiar, a finales de la década de 1980 e inicios de 1990. No obstante, a partir del año 2000, las diversas instituciones y organizaciones del ámbito nacional encargadas de potencializar actividades y sectores promisorios para el desarrollo social y económico del país ejecutaron diferentes programas con este fin.

En esta misma lógica, dichas empresas aprovecharon algunos beneficios que le propició el Estado por medio de sus entidades. Los beneficios obtenidos ICR ${ }^{1}$ por un lado constituyeron un excelente aliciente para continuar fortaleciendo las empresas y, por otro, los continuos contratos de aprendices de SENA ${ }^{2}$, de los que se sirvieron estas organizaciones en su consolidación.

De otra parte, sobresale el hecho de que los empresarios se encuentran fuertemente relacionados con entidades que agremian empresas del sector. Por ejemplo, para tomar un caso, un empresario local ejerce como director de Fedeacua ${ }^{3}$, la organización gremial más poderosa a nivel nacional en el ramo piscícola, desde donde se impulsan proyectos de desarrollo y fortalecimiento de las empresas dedicadas al negocio.

Así mismo, el apoyo del Estado es notable en los procesos de transferencia tecnológica, hace presencia la figura de cooperación técnica en tecnología blanda en forma de asesoría, investigación y adiestramiento (actividades intelectuales); cooperación básicamente hecha a través del patrocinio de seminarios, viajes técnicos, asesorías de plazo limitado, promoción de cursos, asignación de becas y otras formas de actividades estrictamente intelectuales (Rodríguez y Cordero; 2002: 231) realizadas con asociaciones gremiales de la actividad 0 entidades estatales del orden descentralizado.

Despuntan el apoyo brindado por Fedeacua y Acuapez $^{4}$, en los procesos de vigilancia tecnológica. Ca-

1. El Incentivo a la Capitalización Rural (ICR), es un beneficio económico que se otorga a una persona natural o jurídica que en forma individual o colectiva, ejecute un proyecto de inversión nueva, con la finalidad de mejorar la competitividad y sostenibilidad de la producción. Este incentivo consiste en un abono que realiza Finagro al saldo del crédito contraído por el beneficiario para financiar las actividades de inversión objeto del incentivo.

2. Los empleadores podrán deducir anualmente de su renta gravable, hasta el $130 \%$ de los gastos por salarios y prestaciones sociales de los trabajadores contratados como aprendices, adicionales a los previstos legalmente, en programas de formación profesional previamente aprobados por el Servicio Nacional de Aprendizaje SENA.

3. Federación Colombiana de Acuicultores (Fedeacua).

4. La Corporación Centro de Desarrollo Tecnológico Piscícola Surcolombiano-ACUAPEZ es una entidad de participación mixta, de derecho privado, de carácter científico y técnico sin fines de lucro, cuyo objeto es el desarrollo y la ejecución de la investigación, la innovación de la piscicultura, la gestión y la transferencia de la tecnología, mediante la generación y fortalecimiento de la capacidad de innovación tecnológica y desarrollo científico como uno de los factores que determinen la consolidación de la cadena piscícola en el Departamento del Huila. 
bría afirmar, a riesgo de incurrir en exactitudes, que las empresas en mención, junto con otras de la actividad, soportan algunos procesos de vigilancia y captura tecnológica en las entidades gremiales mencionadas.

Es preciso advertir que Acuapez es una empresa de economía mixta que tiene como función aunar esfuerzos y estrechar vínculos para la conformación de sinergias «Universidad - Empresa - ACUAPEZ - Estado» entre el sector Público - Sector Académico - Sector productivo, para la realización de acciones que permitan presentar soluciones viables para el sector, mejorar los niveles de productividad, competitividad y sostenibilidad de la piscicultura regional; propendiendo por el uso de los recursos financieros, físicos, ambientales y humanos ${ }^{5}$.

La expedición de los Decretos ley 393 y 591 de 1991, reglamentarios de la Ley 29 de 1990, permitió que el sector se viera ampliamente beneficiado al contar con herramientas jurídicas para celebrar contratos y convenios para el desarrollo tecnológicos de las empresas. Al respecto su artículo 8 dice:

«La Nación sus entidades descentralizadas podrán celebrar contratos de financiamiento destinados a actividades científicas y tecnológicas, que tengan por objeto proveer de recursos al particular contratista... (Decreto 591/1991)».

Así mismo esta legislación permitió la destinación de recursos para la realización de proyectos de transferencia tecnológica para las empresas del sector, financiados en gran parte con la hacienda pública. En efecto, existen sendos ejemplos como las plantas de procesamiento de algunas empresas fueron beneficiadas mediante convenios de investigación tecnológica (Seilam Ltda -Sena) en buenas prácticas de manufactura, implementación de sistemas de gestión de calidad y aseguramiento en los procesos agroindustriales.

\section{Conclusiones}

En conclusión una de las aparentes causas del surgimiento de las actividades económicas mencionadas fue la existencia de oportunidades ya identificadas. Uno de los factores claves señalados por Valdaliso et al. (2000), como el crecimiento de los mercados.
Tal como lo afirma para el caso de los dos sectores es claro que su origen está fuertemente ligado a la existencia de oportunidades detectadas. Para los dos casos, los ambientes de oportunidades, en mayor medida para la agroindustria por sus niveles de beneficio, fueron generados por el Estado.

Para sintetizar, el surgimiento de la explotación minera como actividad económica en el departamento del Huila se dio bajo el siguiente esquema (ver gráfico 1). El punto de partida lo constituyó, como menciona Valdaliso et al. (2000), la presencia de un mercado consolidado que demandaba constantemente este tipo de productos, ubicado en ciudades como Bogotá, Cali y Medellín.

En esta lógica, agentes empresariales que se desenvolvían dentro de este entorno, percibiendo las oportunidades que le ofrecía el mercado referenciado, deciden aprovechar los beneficios que entregaba. Por lo general, estos agentes eran foráneos pertenecientes a otras regiones del país. Para el fenómeno local, sobresalen algunos casos de individuos venidos de ciudades como Ibagué y Bogotá.

Posteriormente, conocedores del mercado que demandaba este tipo de productos y soportados en información técnica sobre recursos calcáreos y marmóreos con amplia presencia en el departamento, en gran medida suministrada por el Estado a través de los diferentes estudios que se propiciaron a partir de la segunda mitad del siglo XX, permitieron consolidar una oportunidad de negocio.

Frente a los capitales que se utilizaron en estos primeros momentos, se deduce que agentes foráneos no poseían capitales notables para la inversión en el negocio. Su mayor activo lo constituyó de alguna manera el conocimiento del mercado y contactos con posibles compradores para la venta del producto.

En efecto, el actuar de estos agentes comprendió el vínculo con propietarios de terrenos en los cuales se asentaban las reservas del mineral, y ocasionalmente con agentes locales, para la explotación. Fueron precisamente los propietarios y agentes locales los que realizaron las pocas inversiones que requería la precaria explotación. Sin embargo, el manejo del negocio por lo general recaía en manos de los foráneos.

5. http://www.acuapezcdt.org/index.php?option=com_content\&view=article\&id=1\&ltemid=2 

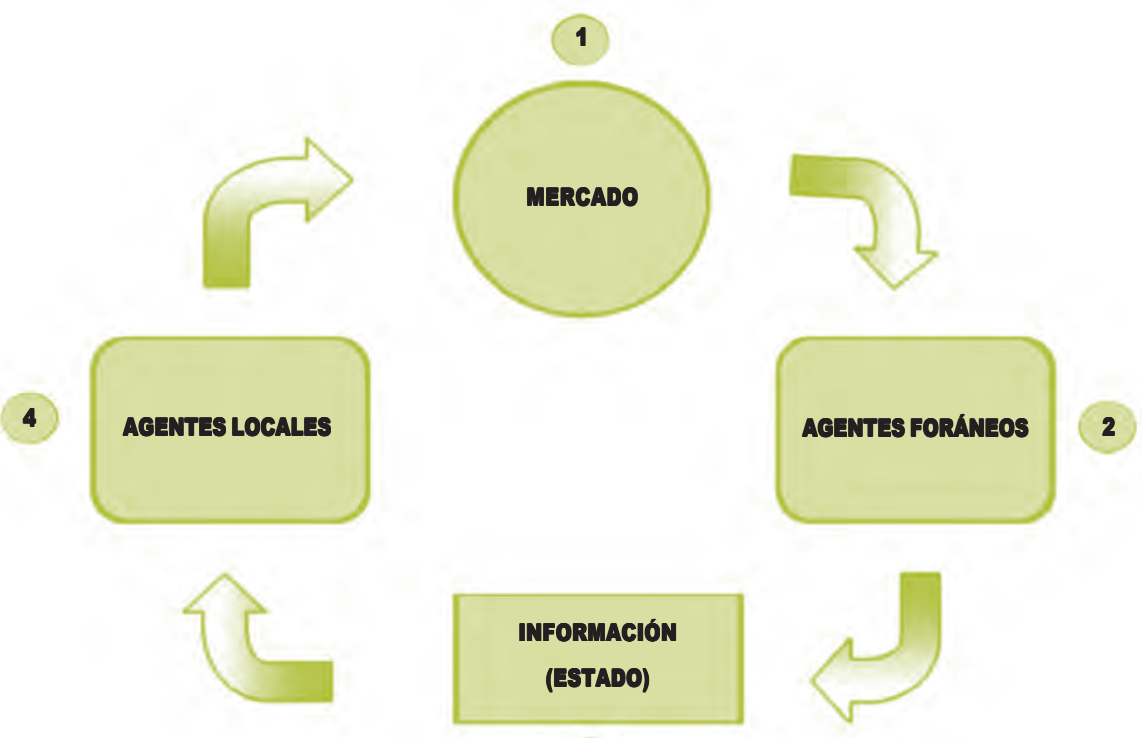

3

Gráfico 1. Esquema actividad minera.

Con el paso del tiempo, estos últimos se retiraron de la actividad dejando a sus socios locales convertidos en mineros, con alguna experiencia en el proceso de explotación y transformación básica, y con contactos en ciudades capitales importantes. Además del reconocimiento hacia afuera que adquirió el Huila como productor del mineral, en aquel momento.

La situación descrita se podría asemejar a una escuela en la cual los agentes locales adquirieron ciertas habilidades y destrezas que permitieron posteriormente el desarrollo, a pesar de lo incipiente de la actividad, con el surgimiento de empresas extractivas y transformadoras.

Para el sector agroindustrial, se pueden identificar tres momentos 0 eslabones que jugaron un papel fundamental en el surgimiento de la actividad económica (ver gráfico 2). Al igual que para el caso anterior, había un precedente de existencia de un mercado nacional e internacional y en su defecto, una demanda establecida.

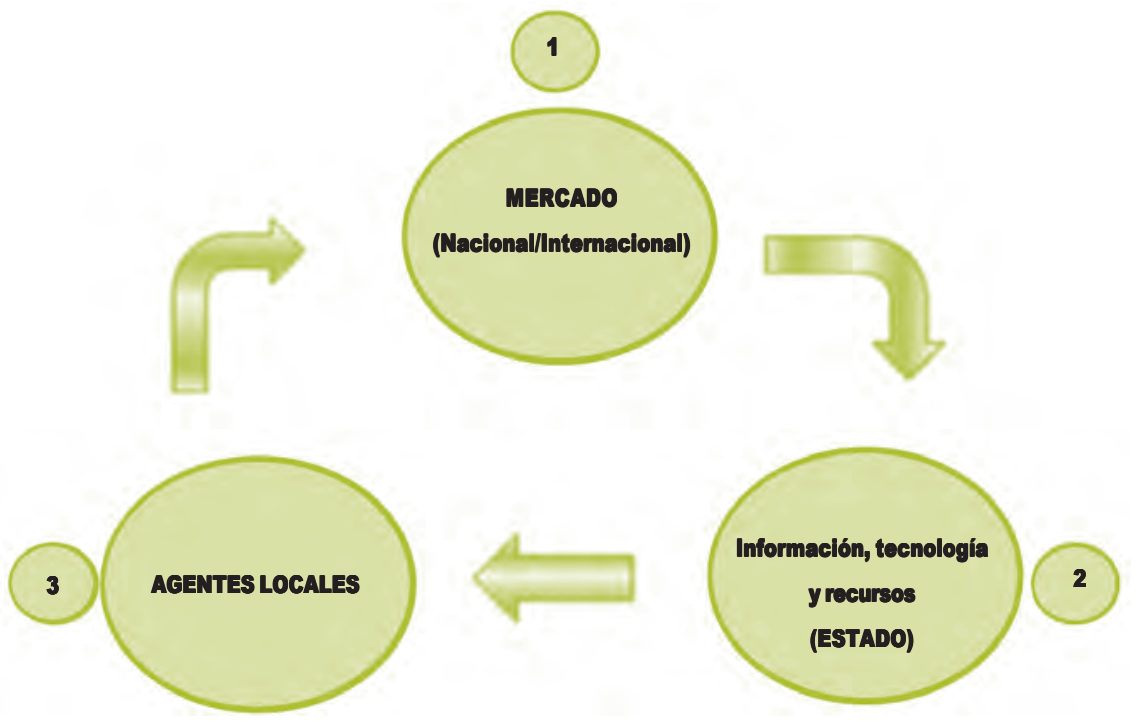

Gráfico 2. Esquema actividad agroindustrial. 
Se puede entonces identificar como punto de partida el mercado nacional e internacional, el cual es identificado por un fuerte nivel de demanda.

Esta demanda que se presenta es jalonada por el consumo de ciudades importantes como Bogotá, Cali y Medellín que para el final de la década de 1990, eran los centros urbanos con grandes consumos por excelencia, donde la oferta era ampliamente superada y se presentaba una necesidad de productos.

A partir de allí, el Estado empieza a generar condiciones benéficas en el ambiente a la aparición y crecimiento de empresas ligadas a procesos agroindustriales que produjeran productos de este tipo.

Aspectos como programas de desarrollo, leyes articuladoras y financiadoras de las empresas a procesos de desarrollos tecnológicos, proyectos de subsidio sobre inversiones, estudios técnicos de producción y explotación, fueron las herramientas y los medios utilizados por las instituciones del Estado para dicho propósito.

El Estado motivó a que las iniciativas locales tuviesen lugar y que a partir de la segunda mitad de 1990 e inicios del 2000, surgieran muchas empresas agroindustriales que posteriormente ubicaron al departamento como primer productor en muchos productos.

De tal suerte, que finalmente los agentes empresariales locales, conocedores de mercados insatisfechos y detectando una serie de condiciones favorables en el ambiente, deciden aventurarse destinando modestas sumas de capital a dichos negocios. Posteriormente, las instituciones Estatales financian procesos de modernización y adecuación de maquinaria y equipos, especialmente las plantas de procesamiento y producción.

Además, realizando ambiciosos programas de promoción internacional y ruedas de negocios, junto con viajes y seminarios internacionales y la vinculación de expertos internacionales para proyectos locales, generó muchos alicientes al sector.

Finalmente se puede deducir que el Estado jugó un papel fundamental en el surgimiento de los dos sectores económicos, y el nivel y las características del desarrollo dependen en gran medida del nivel de intervención e influencia que realizó. Al respecto, el sector minero se encuentra más retrasado en términos tecno- lógicos, organizacionales y de mercado que el agroindustrial.

En este último, se puede apreciar más la disposición de recursos y esfuerzos institucionales para estimular su desarrollo.

\section{Referencias bibliográficas}

Asesorías y Servicios Agroindustriales ASERAGRO Ltda. «Estudio de factibilidad para la conformación de una comercializadora de la producción de materiales pétreos provenientes del distrito minero de Palermo y sus derivados industriales de origen mineral, en el departamento del Huila», 2005.

Dávila L. de Guevara, Carlos. Empresas y Empresarios en la Historia de Colombia. Siglos XIX-XX. Una colección de estudios recientes. Bogotá: Ediciones Uniandes y Grupo Editorial Norma, 2003.

Empresa e historia en América Latina, un balance historiográfico. Bogotá: TM editores-Colciencias, 1996.

Historia empresarial de Colombia, estudios, problemas y perspectivas. Bogotá: Universidad de los Andes, 1991.

El empresario colombiano, una perspectiva histórica. Bogotá: Pontificia Universidad Javeriana, Facultad de estudios interdisciplinarios, 1986.

Doner, Richard. Driving a Bargain. Berkeley, University of California Press, 1991.

Ducuara, Alberto y Manrique, Alfonso. Dimensión histórica de la agroindustria arrocera: Una reflexión empresarial para el Huila 1930-1990. Neiva: Editorial Universidad Surcolombiana, 2008.

Estudios Técnicos Ltda. Estudio de factibilidad técnica para una fábrica de cemento Pórtland en el municipio de Timaná. Informe Final, 1983.

Evans, Peter. Embedded Autonomy: States and Industrial Transformation, Princeton, Princeton University Press, 1995.

Lasso G., Luis Miguel y Sánchez M., Marcos. Industria marmolera. Universidad del Tolima, 2010. 
North, Douglass C. The New Institutional Economics And Development. American Economist, 1987.

Protexa S.A. Estudio de Factibilidad para la Implementación de una Fábrica de cementos Pórtland en el Departamento del Huila, 1967.

Quintero Bonilla, Alexander y Centeno Tapiero, Rolando. Hacendados, comerciantes y negociantes de Neiva a finales del siglo XIX. Neiva: Universidad Surcolombiana, 2005.

Rodríguez Becerra, Manuel. El empresario industrial del viejo Caldas. Bogotá: Universidad de Los Andes, 1993.
Silva Colmenares, Julio. Colombia: crisis del crecimiento económico. Expresión en el comportamiento de la oferta final 1990-2002. Economía y Desarrollo- Septiembre, vol. 2, n 2. Universidad Autónoma de Colombia. En: http://www. fuac.edu.co/revista/////dos.pdf, 2003.

Timmons, Jeffy A. La mentalidad empresarial. Argentina: Editorial Suramericana, 1989.

Valdaliso, Jesús M. y López, Santiago. Historia económica de la empresa, Barcelona, Crítica, 2000. 